\title{
A continuous common-source outbreak of campylobacteriosis associated with changes to the preparation of chicken liver pâté
}

\author{
M. C. O’LEARY ${ }^{1,2 *}$, O. HARDING ${ }^{3}$, L. FISHER ${ }^{4}$ AND J. COWDEN ${ }^{2}$ \\ ${ }^{1}$ European Programme for Intervention Epidemiology Training (EPIET), Stockholm, Sweden \\ ${ }^{2}$ Health Protection Scotland, Clifton House, Clifton Place, Glasgow, Scotland, UK \\ ${ }^{3}$ NHS Forth Valley, Stirling, Scotland, UK \\ ${ }^{4}$ Stirling Council, Environmental Health Department, Stirling, Scotland, UK
}

(Accepted 10 June 2008; first published online 23 July 2008)

\section{SUMMARY}

In December 2006 an outbreak of Campylobacter infection occurred in Forth Valley, Scotland, affecting 48 people over a 3-week period. All cases dined at restaurant A. We conducted a cohort study in a party of 30 who ate lunch at restaurant A on 21 December to identify the vehicle of infection. Of 29 respondents, the attack rate in those who ate chicken liver pâté was $86 \%(6 / 7)$ compared to $0 \%(0 / 22)$ for those who did not. Between 1 December and 1.30 p.m. on 21 December the restaurant had used a different method of cooking the pâté. No cases reported dining at the restaurant after this time. The outbreak's duration suggested a continuous source. This is the first continuous source outbreak of Campylobacter documented in Scotland. Chicken liver pâte was the most likely vehicle of infection. This outbreak illustrates the hazards associated with undercooking Campylobacter-contaminated food.

Key words: Continuous, Campylobacter, foodborne, outbreak, source.

\section{INTRODUCTION}

Campylobacter is the most common laboratoryidentified cause of infectious intestinal disease in humans in Scotland. About 5000 cases are reported each year, with an incidence in 2006 of $95 \cdot 3 / 100000$ population [1]. Despite its high incidence, only four outbreaks were reported between 2002 and 2006, all occurring in 2005.

Symptoms of campylobacteriosis include diarrhoea, bloody diarrhoea, vomiting, nausea and fever. Occasionally infection leads to severe sequelae such as Guillain-Barré syndrome and reactive arthritis. The

\footnotetext{
* Author for correspondence: M. C. O'Leary, Health Protection Scotland, Clifton House, Clifton Place, Glasgow G3 7LN, Scotland, UK.

(Email: maureen.oleary@hps.scot.nhs.uk)
}

incubation period is usually between 2 and 4 days and seldom outside the range of 1-7 days. Symptoms typically last from 1 day to 1 week but can persist for much longer [2].

Consumption of contaminated water and food, such as undercooked meats, and in particular undercooked poultry, is a common cause of infection in humans. Vehicles including milk, fatty foods and water may facilitate the transfer of the bacterium through the gastric acid barrier and so allow some infections to occur at a relatively low infectious dose [2]. Continuous common-source outbreaks are usually associated with contaminated water supplies [3, 4]. Only one foodborne continuous common-source outbreak of campylobacteriosis, due to the distribution of contaminated chicken from a single poultry producer, has been reported in the literature [5]. 
Between 15 and 21 December 2006 Forth Valley National Health Service Board (FV NHSB) received four laboratory reports of cases of Campylobacter. As per standard practice, environmental health officers (EHOs) interviewed all cases using a standard surveillance questionnaire. As all four cases had recently eaten chicken liver pâté at restaurant $\mathrm{A}$, part of a chain of three restaurants in the area with a common owner, EHOs reported the suspected outbreak to the on-call Consultant in Public Health Medicine (CPHM), who on 4 January 2007 convened an outbreak control team (OCT) to investigate and manage the outbreak. The objectives of the outbreak investigation were to describe the extent of the outbreak, to identify its cause and to identify appropriate control measures to help prevent further cases and similar outbreaks in the future.

\section{MATERIALS AND METHODS}

\section{Descriptive epidemiology}

A probable case was defined as a person with at least one of the following symptoms: diarrhoea $(\geqslant 3$ loose stools in $24 \mathrm{~h}$ ), vomiting, stomach pains or bloody stools, with a date of onset of illness after 1 December 2006, who had eaten at one of three restaurants in the area with a common owner. A confirmed case was a probable case who had Campylobacter cultured from their stool.

The CPHM alerted general practitioners and accident and emergency units in the area to the outbreak. The OCT ascertained additional cases through the existing passive surveillance systems such as the notification of food poisoning and the laboratory reporting system for Campylobacter. Additional cases self-reported to the local council. Some self-reported cases also reported illness among their colleagues. EHOs traced contacts of the self-reported cases.

EHOs compiled a list of all laboratory-reported cases and took their food history using the standardized surveillance questionnaire. A full food history was not taken from self-reported cases or cases indicated by them, however, they were asked if and when they had dined at restaurant A. This was conducted prior to the instigation of the outbreak investigation.

In the last 2 weeks of January EHOs telephoned all the cases documented as being part of the outbreak and not included in the subsequent analytical study and obtained data on each case including: gender, date of birth, date of visiting the restaurant, date of onset of symptoms, whether they had submitted a specimen, whether the specimen was positive and whether they had consumed chicken liver pâté.

\section{Analytical epidemiology}

We conducted a retrospective cohort study among a single party of 30 people who ate lunch at restaurant A on 21 December and who reported illness in their group, to test the hypothesis that chicken liver pâté prepared at restaurant A was the outbreak's vehicle of infection. This party represented a convenient well-defined cohort from whom it was relatively easy to collect data and they were one of the most recent groups reporting illness having dined at restaurant A. The cohort did not include cases used in hypothesis generation. EHOs interviewed the cohort between 5 and 22 January 2007 and collected information on all menu items consumed at restaurant A using a standardized questionnaire. EHOs asked all respondents to submit a stool specimen for analysis if they had not already done so.

\section{Data analysis}

We analysed data using EpiData Analysis (version 1.1; EpiData Association, Odense, Denmark) and R (version 2.4.1; R Foundation for Statistical Computing, Vienna, Austria). We calculated food specific attack rates, relative risks (RR) and $95 \%$ confidence intervals (CI). For those food items where the RR was infinite and where EpiData Analysis could not calculate a $95 \% \mathrm{CI}$, the $95 \% \mathrm{CI}$ was calculated using the Cox-Hinkley-Miettinen-Nurminen method [6]. We conducted an adjustment by adding a value of 1 to each cell in the $2 \times 2$ table to allow us to estimate the $\mathrm{RR}$ for food items where no non-cases consumed the food. We used Fisher's exact test to determine statistical significance.

\section{Risk assessment}

EHOs visited restaurant A twice between 19 and 21 December. On the second visit they observed that the chicken liver pâté had a pinker appearance than usual and questioned staff about the production method. A sample of about $2 \mathrm{~kg}$ of pâte that was being served at the restaurant on that day was taken for microbiological culture. No other foods were sampled. 


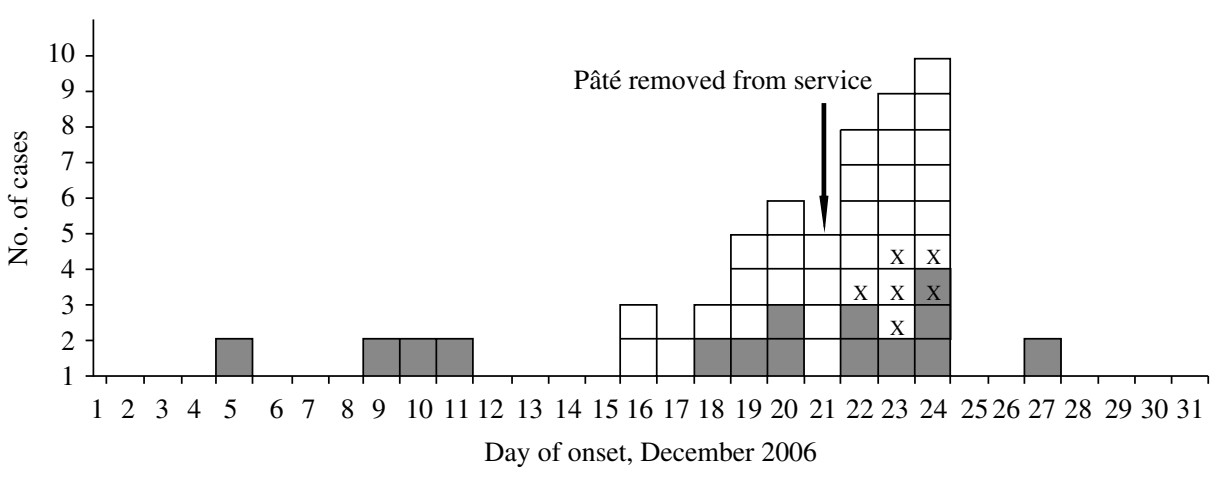

Fig. 1. Number of known confirmed $(\square)$ and probable $(\square)$ cases of Campylobacter gastroenteritis associated with dining at restaurant A $(n=47)$, by date of onset of symptoms, December 2006, Forth Valley NHS Board, Scotland. X indicates a case included in the cohort study (one case with missing information on date of onset not included).

The pâté sample was sent to Glasgow Scientific Services, where there was a 13-day delay before culturing it. Thirty grams of pâté were cultured by pre-enrichment in Bolton Broth under microaerophilic conditions for $4 \mathrm{~h}$ at $37{ }^{\circ} \mathrm{C}$ followed by $44 \mathrm{~h}$ at $41.5{ }^{\circ} \mathrm{C}$ and subsequent culture on solid selective media under microaerophilic conditions at $41.5^{\circ} \mathrm{C}$ for $48 \mathrm{~h}$. Suspect colonies were confirmed using appropriate biochemical tests.

\section{RESULTS}

By 19 January 2007 a total of 48 cases were identified (15 confirmed and 33 probable) (Fig. 1). Two of these were hospitalized. There was a median of 2 days between onset of symptoms and dining at restaurant A (range $0-9$ days). Of the 48 cases, 38 (79\%) were female. Of the 32 cases for whom age was known, the median age was 38 years (range 23-64 years). All cases reported dining at the restaurant between 3 and 21 December 2006 and all reported consuming chicken liver pâté. Using the chef's estimate of about 53 portions of pâte having been served during this period, the attack rate amongst those who consumed pâté was $91 \%(48 / 53)$. This is, however, speculative and cannot be verified. Eleven Christmas work parties were affected by the outbreak accounting for 34 of the cases. The attack rates for four parties were known $(10 \%, 20 \%, 21 \%$ and $32 \%)$.

The response rate for the cohort study was $97 \%$ (29/30) including one confirmed and five probable cases (attack rate $21 \%$ ). The attack rate for females (26.3\%) was higher than for males (10\%) (RR 2.63, $95 \%$ CI 0.4-17.0). The median age of the participants was 39 years (range 20-58 years). One case did not report their age. Of the remaining cases, the median age was 31 years (range 22-47 years). All other data collected was complete. Among cases the mean duration of illness was 11 days (range 6-15 days), however, two respondents were still symptomatic at the time of interview.

The attack rate among those who ate pâté was $85.7 \%(6 / 7)$ compared to $0 \%(0 / 22)$ for those who did not (RR $\infty, 95 \%$ CI $4.9-\infty, P<0 \cdot 0001$ ) (Table 1 ). If there had been a single case amongst those who did not consume pâté the RR would have been 18.7 (95\% CI $2 \cdot 7-131 \cdot 2, P=0 \cdot 0001)$. The attack rate was $100 \%$ (2/2) among those who ate salmon compared to $14.8 \%(4 / 27)$ among those who did not (RR 6.8, $95 \%$ CI $2 \cdot 7-16 \cdot 7, P=0 \cdot 04)$. No other food items were associated with illness. We excluded food items that were not eaten by the cohort.

Fifteen of $21(71 \%)$ cases who submitted specimens tested positive for Campylobacter. Campylobacter was not cultured from the pâté. A new method of cooking pâté was introduced on 1 December involving lightly sautéing fresh chicken livers until coloured on the outside followed by blending them with the other ingredients. The new method was instigated to produce a pinker more aesthetically pleasing product. At around 1.30 p.m. on 21 December the chef agreed to remove the pâté from the menu, although pâté was still served at lunch that day. No cases reported dining at the restaurant after lunchtime on this date.

\section{DISCUSSION}

We report a continuous common-source outbreak whereby the descriptive epidemiology, the observations made during the risk assessment and the results of the cohort study suggest that chicken liver pâté was the most probable vehicle of infection. 
Table 1. Food specific attack rates $(A R)$, relative risks $(R R), 95 \%$ confidence intervals $(C I)$ and Fisher's exact test $P$ values, outbreak of Campylobacter, restaurant A, Scotland, December 2006

\begin{tabular}{|c|c|c|c|c|c|c|c|c|c|}
\hline \multirow[b]{2}{*}{ Food } & \multicolumn{3}{|c|}{ Food eaten } & \multicolumn{3}{|c|}{ Food not eaten } & \multirow[b]{2}{*}{$\mathrm{RR}$} & \multirow[b]{2}{*}{$95 \% \mathrm{CI}$} & \multirow{2}{*}{$\begin{array}{l}\text { Fisher's exact } \\
\text { test } P \text { value }\end{array}$} \\
\hline & Cases & Total & $\operatorname{AR}(\%)$ & Cases & Total & $\operatorname{AR}(\%)$ & & & \\
\hline Chicken liver pâté & 6 & 7 & $85 \cdot 7$ & 0 & 22 & 0 & $\infty$ & $4 \cdot 9-\infty$ & $<0.0001$ \\
\hline Chicken liver pâté* & 7 & 8 & $87 \cdot 5$ & 1 & 23 & $4 \cdot 3$ & $18 \cdot 7$ & $2 \cdot 7-131 \cdot 2$ & $0 \cdot 0001$ \\
\hline Salmon & 2 & 2 & $100 \cdot 0$ & 4 & 27 & $14 \cdot 8$ & $6 \cdot 8$ & $2 \cdot 7-16 \cdot 7$ & $0 \cdot 0369$ \\
\hline $\begin{array}{l}\text { Cherry \& } \\
\text { almond tart }\end{array}$ & 3 & 10 & $30 \cdot 0$ & 3 & 19 & $15 \cdot 8$ & 1.9 & $0 \cdot 5-7 \cdot 8$ & $0 \cdot 6328$ \\
\hline Tiramisu & 2 & 7 & $28 \cdot 6$ & 4 & 22 & $18 \cdot 2$ & $1 \cdot 6$ & $0 \cdot 4-6 \cdot 8$ & $0 \cdot 6199$ \\
\hline Christmas pudding & 1 & 5 & $20 \cdot 0$ & 5 & 24 & $20 \cdot 8$ & $1 \cdot 0$ & $0 \cdot 1-6 \cdot 5$ & $1 \cdot 0000$ \\
\hline Turkey & 3 & 15 & $20 \cdot 0$ & 3 & 14 & $21 \cdot 4$ & $0 \cdot 9$ & $0 \cdot 22-3 \cdot 9$ & $1 \cdot 0000$ \\
\hline Steak & 1 & 6 & $16 \cdot 7$ & 5 & 23 & $21 \cdot 7$ & $0 \cdot 8$ & $0 \cdot 1-5 \cdot 4$ & $1 \cdot 0000$ \\
\hline Cheese board & 0 & 4 & $0 \cdot 0$ & 6 & 25 & $24 \cdot 0$ & $0 \cdot 0$ & $0 \cdot 0-2 \cdot 5$ & $0 \cdot 5526$ \\
\hline Chicken & 0 & 2 & $0 \cdot 0$ & 6 & 27 & $22 \cdot 2$ & $0 \cdot 0$ & $0 \cdot 0-4 \cdot 0$ & $1 \cdot 0000$ \\
\hline $\begin{array}{l}\text { French onion } \\
\text { soup }\end{array}$ & 0 & 1 & $0 \cdot 0$ & 6 & 28 & $21 \cdot 4$ & $0 \cdot 0$ & $0 \cdot 0-5 \cdot 3$ & $1 \cdot 0000$ \\
\hline Fried rice & 0 & 1 & $0 \cdot 0$ & 6 & 28 & $21 \cdot 4$ & $0 \cdot 0$ & $0 \cdot 0-5 \cdot 3$ & $1 \cdot 0000$ \\
\hline Melon & 0 & 7 & $0 \cdot 0$ & 6 & 22 & $27 \cdot 3$ & $0 \cdot 0$ & $0 \cdot 0-1 \cdot 5$ & $0 \cdot 2885$ \\
\hline $\begin{array}{l}\text { Mushroom \& ham } \\
\text { croquette }\end{array}$ & 0 & 5 & $0 \cdot 0$ & 6 & 24 & $25 \cdot 0$ & $0 \cdot 0$ & $0 \cdot 0-2 \cdot 1$ & $0 \cdot 5526$ \\
\hline Risotto & 0 & 3 & $0 \cdot 0$ & 6 & 26 & $23 \cdot 1$ & $0 \cdot 0$ & $0 \cdot 0-3 \cdot 1$ & $1 \cdot 0000$ \\
\hline $\begin{array}{l}\text { Salmon \& } \\
\text { prawns }\end{array}$ & 0 & 5 & $0 \cdot 0$ & 6 & 24 & $25 \cdot 0$ & $0 \cdot 0$ & $0 \cdot 0-2 \cdot 1$ & $0 \cdot 5526$ \\
\hline $\begin{array}{l}\text { Soup of the } \\
\text { day }\end{array}$ & 0 & 3 & $0 \cdot 0$ & 6 & 26 & $23 \cdot 1$ & $0 \cdot 0$ & $0 \cdot 0-3 \cdot 1$ & $1 \cdot 0000$ \\
\hline
\end{tabular}

* Adjusted analysis.

Changes made to its preparation on 1 December led to the production of several undercooked batches that resulted in an outbreak of campylobacteriosis that affected 48 people between 3 December and lunchtime on 21 December.

Our investigation had a number of limitations. Chicken liver pâté was the only food item sampled and Campylobacter was not successfully cultured from it.

Campylobacter is sensitive to drying and oxygen, although it survives well at temperatures as low as $4{ }^{\circ} \mathrm{C}$ [7]. A cross-section of the pâté, that included material from the core, where lower oxygen levels were expected, was cultured. A 13-day delay in processing the sample, over the Christmas holidays, may have adversely impacted on the potential to successfully culture Campylobacter from it, despite its storage under favourable conditions and despite the inclusion of an enrichment step in the culturing procedure.

Only one of four listed symptoms of campylobacteriosis was needed to meet the probable case definition. This may have resulted in the misclassification of non-cases as cases. However, all but five cases reported two or more symptoms of infection including all cases in the cohort study.

EHOs took a full food history from all laboratoryconfirmed cases, however, when they interviewed probable cases as part of the descriptive study, they specifically enquired about the consumption of pâté, possibly leading to a biased response and overreporting of pâté consumption. The use of different subjects in the cohort study, which sought information on all menu items, ensured that bias during hypothesis testing was minimized. However, as members of the cohort were not interviewed until 2-3 weeks after dining at the restaurant, poor recall may have limited the accuracy of response.

Since the outbreak appeared to be over by the time the analytical investigation was instigated, a small cohort study with a single party of diners was considered to be the best use of resources. This limited statistical power and included only a fraction of the cases documented in the outbreak. Despite this a statistically significant higher attack rate was detected among those who ate pâté compared to those who did not. 
The attack rate and RR for salmon was also high, however, the only two people who ate salmon also ate pâté making it impossible to conduct a stratified analysis. We consider the association between eating salmon and illness to be due to confounding for several reasons. Salmon is not commonly contaminated with Campylobacter and although its high fat content makes it a plausible vehicle of infection, it is rarely cited as such. Moreover, Campylobacter would have been destroyed during cooking, although crosscontamination following cooking is a possibility. Last, if salmon were the only vehicle of infection, the four cases who did not eat salmon could not be explained.

Campylobacter has been found in over $75 \%$ of raw chicken on sale in Scotland [8]. As chicken liver pâté is a fatty food, it may favour the passage of Campylobacter through the gastric acid barrier [2]. The survival of even small numbers of Campylobacter in the pâté would be sufficient to cause illness.

Campylobacter can infect both the external and internal tissue of chicken livers $[9,10]$. One study reported the isolation of Campylobacter from $26 \%$ of surface-sterilized livers [9], while another reported its isolation from $100 \%$ of chicken liver surfaces and, following surface sterilization, $90 \%$ of interiors, at densities of up to $>10^{4}$ MPN (most probable number) per liver [10]. The authors demonstrated that inactivation of Campylobacter is proportional to cooking time. With pan-frying, core temperatures stabilized at $70-80^{\circ} \mathrm{C}$ after $2.5 \mathrm{~min}$ and Campylobacter was not completely inactivated until after 5 min. Livers remained bloody until after 3 min of cooking time and remained pink until $5 \mathrm{~min}$, after which time they turned grey.

It is likely that the new recipe employed at restaurant A did not ensure sufficiently high internal temperatures to kill all the Campylobacter, particularly if the livers were heavily contaminated.

This is the first foodborne continuous commonsource outbreak of campylobacteriosis reported in Scotland since the instigation of the current national infectious intestinal disease surveillance system in 1996. It highlights the importance of the risk assessment in terms of population health protection. Early identification of unsafe cooking practices, in premises where the overall standard of hygiene was high, led to the implementation of control measures that prevented further cases and successfully limited the outbreak prior to the instigation of the analytical epidemiological investigation.
Following this outbreak the following recommendations were made: chicken liver pâte should be produced using a method that guarantees a product that is consistently cooked throughout, such as blending all ingredients followed by oven baking to a minimum core temperature of $75^{\circ} \mathrm{C}$; changes to recipes should be accompanied by a reassessment of the hazards and implementation of the necessary controls to ensure a safe product.

\section{ACKNOWLEDGEMENTS}

We thank all those involved in the outbreak investigation, particularly the members of the outbreak control team, as well as staff at Health Protection Scotland for statistical and epidemiological support. M. C. O'Leary is a Fellow with the European Programme for Intervention Epidemiology Training.

\section{DECLARATION OF INTEREST}

None.

\section{REFERENCES}

1. Locking M, et al. Gastrointestinal and foodborne infections, Surveillance Report. HPS Weekly Report 2007; 41: 2007/01 (http://www.hps.scot.nhs.uk/ewr/index.aspx). Accessed 29 January 2007.

2. Blaser MJ. Campylobacter jejuni and related species. In: Mandell GL, Bennett JE, Dolin R, eds. Mandell, Douglas, and Bennett's Principles and Practice of Infectious Diseases, 5th edn. Philadelphia: Churchill Livingston, 2000, pp. 2276-2285.

3. Hänninen ML, et al. Detection and typing of Campylobacter jejuni and Campylobacter coli and analysis of indicator organisms in three waterborne outbreaks in Finland. Applied Environmental Microbiology 2003; 69: 1391-1396.

4. Engberg J, et al. Water-borne Campylobacter jejuni infection in a Danish town - a 6-week continuous source outbreak. Clinical Microbiology and Infection 1998; 4: 648-656.

5. Pearson AD, et al. Continuous source outbreak of campylobacteriosis traced to chicken. Journal of Food Protection 2000; 63: 309-314.

6. Cardiff University School of Medicine, Department of Primary Care and Public Health. Confidence intervals for proportions and related quantities: odds ratio and relative risk calculator (http://www.cardiff.ac.uk/medic/ aboutus/departments/primarycareandpublichealth/ resources/ODDSRATIOANDRR.xls). Accessed 10 February 2007.

7. Advisory Committee on the Microbiological Safety of Food 2005. Second report on campylobacter 
(www.food.gov.uk/multimedia/pdfs/acmfscampylobacter. pdf). Accessed 15 August 2007.

8. Food Standards Agency 2003. UK-wide survey of salmonella and campylobacter contamination of fresh and frozen chicken on retail sale (http://www.food.gov.uk/ multimedia/pdfs/campsalmsurvey.pdf). Accessed 12 November 2007.
9. Baumgartner A, et al. Campylobacter contaminations of poultry liver - consequences for food handlers and consumers. Archiv für Lebensmittelhygiene 1995; 46: $1-24$.

10. Whyte R, Hudson JA, Graham C. Campylobacter in chicken livers and their destruction by pan-frying. Letters in Applied Microbiology 2006; 43: 591-595. 